\title{
Predictors of early and late left atrial tachycardia and left atrial flutter after catheter ablation of atrial fibrillation: Long-term follow-up
}

\author{
Maciej Wójcik ${ }^{1,2}$, Alexander Berkowitsch ${ }^{1}$, Sergey Zaltsberg ${ }^{1}$, \\ Christian W. Hamm ${ }^{1,3}$, Heinz F. Pitschner ${ }^{1}$, Malte Kuniss ${ }^{1}$, Thomas Neumann ${ }^{1,3}$ \\ ${ }^{1}$ Department of Cardiology, Kerckhoff Heart and Thorax Center, Bad Nauheim, Germany \\ ${ }^{2}$ Department of Cardiology, Medical University of Lublin, Lublin, Poland \\ ${ }^{3}$ Department of Cardiology, Justus-Liebig University of Giessen, Germany
}

\begin{abstract}
Background: The aim of the study was identification of the predictors of left atrial tachycardia and left atrial flutter (LATAFL) after radiofrequency catheter ablation of atrial fibrillation $(C A A F)$.

Methods: We followed 598 patients (71\% male, 41\% paroxysmal AF; median follow-up: 36 months) after a single step-wise CAAF procedure. The time to first documented LATAFL lasting longer than $30 \mathrm{~s}$, documented in any kind of electrocardiography (ECG), was defined as an end-point.
\end{abstract}

Results: A single CAAF procedure resulted in LATAF in 58 (10\%) patients. Additional lesions were performed in 275 (46\%) patients. Early LATAFL recurrence ( $\leq 3$ months since the index procedure) was observed in 11 (2\%) patients. Late LATAFL (> 3 months) was noted in $47(8 \%)$ patients. The univariate predictors of LATAFL recurrence were: type of AF $(p=0.003)$, the size of $L A(p=0.002)$ and the type of procedure $(p=0.0001)$. The identified single independent predictors of LATAFL recurrence were enlarged $L A(p=0.001)$ and multiple ( $\geq 2)$ additional lesions performed during the index procedure $(p<0.0001)$.

Conclusions: Higher rate of LATAFL recurrence was observed in patients with non-paroxysmal $A F$, enlarged LA and any additional lesions performed. Two independent predictors of $L A T A F L$ recurrence after $C A A F$ were: the enlarged $L A$ and multiple ( $\geq 2)$ additional lesions performed during the index procedure. (Cardiol J 2015; 22, 5: 557-566)

Key words: atrial fibrillation, catheter ablation, left atrial tachycardia, left atrial flutter

\section{Introduction}

Catheter ablation of atrial fibrillation (AF) is a more effective therapeutic option for patients with $\mathrm{AF}$, as compared to antiarrhythmic drugs (AADs) [1]. Multiple approaches for catheter ablation of AF (CAAF) have been developed. The current techniques focus on the elimination of mechanisms involved in the initiation (triggers) and maintenance (substrate) of $\mathrm{AF}[2,3]$. While ablation strategies that target pulmonary veins (PVs) are the cornerstone for most $\mathrm{AF}$ ablation procedures,

Address for correspondence: Prof. Thomas Neumann, Department of Cardiology, Kerckhoff Heart and Thorax Center, Benekestr. 2-8, 61231 Bad Nauheim, Germany, tel: +49 6032/996-2202, fax: +49 6032/996-2298,

e-mail: t.neumann@kerckhoff-klinik.de

Received: 13.05.2015 Accepted: 07.06.2015 
more extensive ablation based on linear lesions or complex fractionated electrograms (CFAE) are approached in patients with long-standing persistent $\mathrm{AF}[3,4]$. Organized left atrial tachycardia and left atrial flutter (LATAFL) are common after CAAF with a reported incidence of $1.2-40 \%$ and the variability in the frequency of occurrence and the mechanism of LATAFL appears to be clearly dependent on the type of ablation procedure used and the extent of the underlying atrial disease [2]. Nevertheless, the predictors of LATAFL after CAAF are under debate [4].

The aim of the study was identification of the predictors of LATAFL after CAAF.

\section{Methods}

\section{Patient characteristics}

We followed 598 patients who had CAAF performed. The inclusion criteria, at the date of the procedure, were as follows: age 18-80 years, symptomatic and drug refractory non-valvular AF, $\mathrm{AF}$ documented in at least 2 electrocardiograms (ECGs) in a 3-month period preceding the ablation procedure and a signed informed consent obtained from the patient. The exclusion criteria included: acute reversible causes for $\mathrm{AF}$, valvular AF, LATAFL documented in at least $1 \mathrm{ECG}$, known bleeding diathesis, intolerance of heparin or oral anticoagulation, left atrial (LA) thrombus, pregnancy or breastfeeding, abuse of drugs or alcohol, New York Heart Association (NYHA) class IV and other severe co-morbidities.

\section{Pre-ablation preparation}

Medical history was obtained during a visit to an outpatient-clinic with a review of the medical records including ECGs and Holter-ECG recordings of AF. The risk of procedure was discussed in detail, and all patients gave their written informed consent. AADs were discontinued at least 3 days before the ablation. Beta-blockers were allowed according to the protocol.

\section{Assessment of the left atrial size}

All patients underwent transthoracic echocardiography to determine LA diameter. The LA size was assessed by measurement of short and long axis in apical 4-chamber view. The normalized left atrial area (NLA), previously shown to be a stronger predictor of outcome than both LA short and long axis diameters, as well as LA area was calculated in each case [5].

\section{NPAF and LATAFL definition}

Non-paroxysmal AF (NPAF) was defined as persistent, long-lasting persistent, or paroxysmal $\mathrm{AF}$ (PAF) in patients with more than $500 \mathrm{~h}$ in symptomatic AF within 3 months prior to admission [5]. Left atrial tachycardia and left atrial flutter were defined as: (1) early LATAFL (occurring within the first 3 months) and (2) late LATAFL (occurring after the first 3 months).

\section{Catheter ablation procedure}

All procedures were performed under conscious sedation and analgesia. In all cases we used bi-plane fluoroscopy set in $60^{\circ} \mathrm{LAO}$ and $30^{\circ} \mathrm{RAO}$ views, respectively. After local anesthesia, a 10- or 20-polar steerable catheter was placed in the coronary sinus. Two transseptal sheets were introduced into the LA. The ablation procedures were performed with a support of a 3-dimensional (3D) electro-anatomical mapping system (Carto/EnSite) or with the previously described [6] high-density mapping catheter, HD Mesh Mapper $^{\text {TM }}$ (HDMM; Bard Electrophysiology, Lowell, MA, USA), placed in an antrum of the PV. A $7 \mathrm{~F}$ ThermoCool catheter (Biosense Webster) with a 3.5-mm irrigated tip was used for the ablation with radiofrequency energy settings chosen between 15 and $35 \mathrm{~W}$ and a cutoff temperature of $42^{\circ} \mathrm{C}$. Ablation of each PV was carried out at the atrial side of the PV. A circumferential ablation line was performed around each $\mathrm{PV}$, overlapping ipsilateral ablation lines.

The first step was the control of PV antrum isolation. In patients with sinus rhythm, the procedure was seized after confirming isolation of all veins, at least $30 \mathrm{~min}$ after last energy application. In patients with $\mathrm{AF}$, when arrhythmia stopped spontaneously during $\mathrm{PV}$ isolation (PVI) at the antrum, the procedure was also aborted after confirming PVI. If AF continues, a stepwise approach including mitral isthmus line (MIL), roof line (RL), ablation of complex fragmented signals (CFAE) and endocardial lesion along coronary sinus (CSL) was implemented at the same procedure. When sinus rhythm could not be obtained during the procedure, cardioversion and re-mapping at sinus rhythm was planned at the end of procedure. The tightness of all linear lesions, respectively to the performed lesions in certain patient, and isolation of PVs and superior vena cava were controlled.

The acute endpoint for the procedure was elimination of $\mathrm{PV}$ antrum potentials and proving electrical isolation (bi-directional block) of PVs, superior vena cava and linear lesions, if performed. 


\section{Post-ablation management}

Intravenous flow of heparin was continued to achieve a partial thromboplastin time of 60-80 s. All patients underwent trans-thoracic echocardiography to exclude pericardial effusion. Oral anticoagulation with coumadin was started 1 day after CAAF, targeting an international normalized ratio of 2.0 to 3.0 .

\section{Follow-up}

After discharge from the hospital, the patients were scheduled for quarterly follow-up (FU) visits. One year after the intervention, FU visits were performed once a year. Seven-day Holter ECG recordings and a 12-channel ECG were obtained during each FU visit. Patients were asked to obtain an ECG, in our institution or outside, when feeling palpitations at times out of Holter-ECG monitoring periods.

\section{Statistical methods}

Study end-point. Time to first documented episode of LATAFL lasting longer than $30 \mathrm{~s}$, documented in any kind of ECG was defined as an index parameter for a failure. Endpoint for analysis was the LATAFL-free survival within a FU time of 60 months.

Statistical analysis. The effect of discrete variables was studied using the Kaplan-Meier survival analysis with log-rank test. The parameters were dichotomized of derived from receiving operator characteristics (ROC)-curve optimal cut-off value, i.e. at the point, where the sum of sensitivity and specificity reached the maximum values. The impact of discrete variables on the outcome was described with positive and negative prediction accuracy and hazard ratio. The continuous data were presented as median and inter-quartile range, and categorical variables as numbers and percentages.

To avoid a potential model over-fitting, only the parameters revealed to be significantly associated with the outcome in univariate analysis were included in the multivariate Cox regression model performed using a step-down procedure. Two-tailed $\mathrm{p}$ values $<0.05$ were considered statistically significant.

\section{Results}

Baseline and procedural characteristics

In this single-center study we included 598 patients (median FU of 36 months) who underwent a single CAAF procedure. The support of $3 \mathrm{D}$
Table 1. Baseline characteristics $(n=598)$.

\begin{tabular}{lcc}
\hline Parameter & N (\%) or median (IQR) \\
\hline Male & 425 & $(71 \%)$ \\
Age [years] & 59 & $(52 ; 65)$ \\
History of AF [years] & 4.4 & $(2.0 ; 8.2)$ \\
Paroxysmal AF & 243 & $(41 \%)$ \\
Non-paroxysmal AF & 355 & $(59 \%)$ \\
Body mass index & 27.4 & $(25.2 ; 30.5)$ \\
Hypertension & 435 & $(73 \%)$ \\
Coronary artery disease & 49 & $(8 \%)$ \\
Dilated/hypertrophic & 26 & $(4 \%)$ \\
cardiomyopathy & & \\
Diabetes mellitus & 55 & $(9 \%)$ \\
GFR & 86.9 & $(74.7 ; 101.9)$ \\
Metabolic syndrome & 243 & $(41 \%)$ \\
LVEF & 60 & $(57 ; 62)$ \\
LA - short axis & 40 & $(37 ; 43)$ \\
LA - long axis & 55 & $(51 ; 59)$ \\
LA area & 21.8 & $(19.1 ; 25.2)$ \\
Normalized LA area & 10.6 & $(9.3 ; 12.0)$ \\
\hline
\end{tabular}

IOR - inter-quartile range; AF — atrial fibrillation; GFR - glomerular filtration rate; LVEF - left ventricular ejection fraction; LA — left atrium

electro-anatomical mapping system was used in $191(32 \%)$ cases. Patients' baseline characteristics are summarized in Table 1.

Additional lesions were performed in 275 (46\%) patients, including: RL in 220 (37\%), MIL in $198(33 \%)$, CSL in $92(15 \%)$ and CFAE eliminated in $78(13 \%)$ patients. In case of sustained arrhythmia, after having performed all steps of our protocol, the restoration of sinus rhythm with periprocedural direct current electricial cardioversion was finally executed in 169 (28\%) patients.

The median of cumulative time of procedure and fluoroscopy was $4.5(4.0 ; 5.4) \mathrm{h}$ and $61(41 ; 90)$ min, respectively.

\section{LATAFL-free survival and predictors of outcome after a CAAF procedure}

A single CAAF procedure resulted in LATAF in $58(10 \%)$ patients (Fig. 1). Early ( $\leq 3$ months since the index procedure) LATAFL recurrence was observed in $11(2 \%)$ patients. Late $(>3$ months) LATAFL was noted in $47(8 \%)$ patients. The characteristics of both groups are presented in Table 2.

The cut-off value of 11.5 for NLA was calculated from ROC-curve analysis. The univariate 


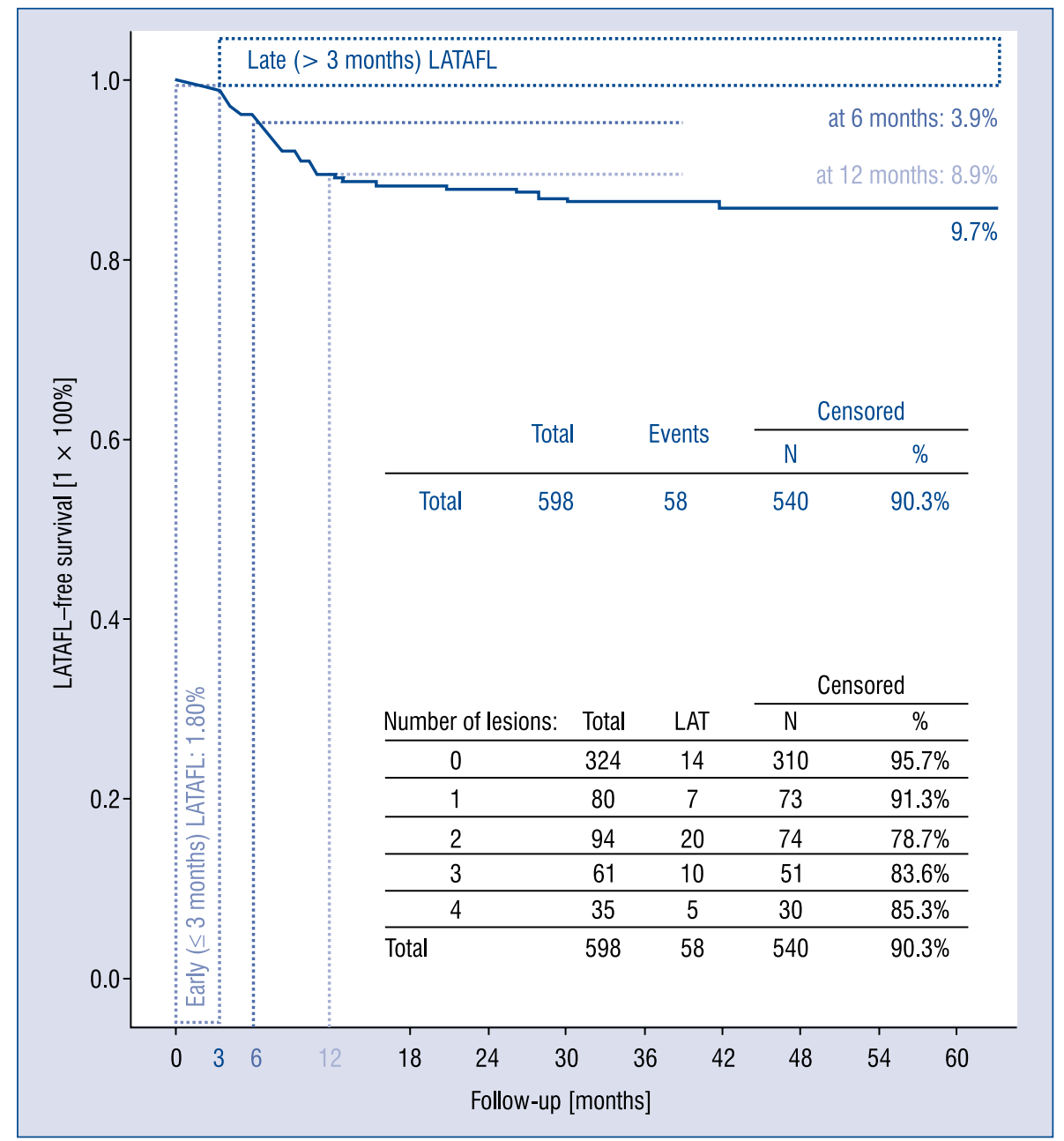

Figure 1. Late left atrial tachycardia and left atrial flutter (LATAFL) and a 5-year outcome. Kaplan-Meier event-free survival curve after a single catheter ablation. We observed recurrences of any arrhythmia (atrial fibrillation/LATAFL) in total of $250(41.8 \%)$ patients during the follow-up period. LATAFL was diagnosed in electrocardiogram in 58 of these 250 patients.

predictors of LATAFL recurrence (Table 3) were: type of AF ( $p=0.003$ ), the size of LA (best defined with NLA, $p=0.002$ ) and the type of procedure $(\mathrm{p}=0.0001)$.

We found that the single independent predictors of LATAFL recurrence were (Table 3) enlarged LA, defined with NLA > 11.5 (Fig. 2A; $\mathrm{p}=0.019)$ and multiple $(\geq 2)$ additional lesions performed during the index procedure (Fig. 2B; $\mathrm{p}<0.0001$ ).

\section{Complications}

Cardiac tamponades, which occurred in $8(1 \%)$ patients, were managed by pericardial puncture without further sequels. Pericardial effusion was found in $13(2 \%)$ patients $(2.17 \%)$.

\section{Discussion}

\section{Main findings}

We describe the independent predictors of LATAFL recurrence after CAAF: enlarged LA and multiple $(\geq 2)$ additional lesions performed during the index procedure.

\section{Outcome}

The present study shows a $10 \%$ LATAFL recurrence rate in 598 patients with non-valvular AF after a single CAAF procedure. We observed most LATAFL recurrence in the first 12 months after CAAF (Fig. 1). The analysis of previous reports [6-29] revealed that total LATAFL recurrence in 5,273 patients after CAAF was $12 \%$. Early and late 
Table 2. The comparison of patients with early and late left atrial tachycardia and left atrial flutter (LATAFL) recurrence in follow-up (FU) $(n=58)$.

\begin{tabular}{|c|c|c|c|c|c|}
\hline & \multicolumn{4}{|c|}{ LAT/LAFL recurrence in FU $-\mathrm{n}(\%)$ or median (IQR) } & \multirow[t]{2}{*}{$\mathbf{P}$} \\
\hline & \multicolumn{2}{|c|}{ Early ( $\leq 3$ months) } & \multicolumn{2}{|c|}{ Late (> 3 months) } & \\
\hline \multicolumn{6}{|l|}{ Baseline data } \\
\hline Number of patients & 11 & & 47 & & \\
\hline Male & 9 & $(82 \%)$ & 33 & $70 \%$ & 0.710 \\
\hline History of AF [years] & 1.38 & $(0.42 ; 2.06)$ & 4.35 & $(1.9 ; 6.84)$ & 0.003 \\
\hline Non-paroxysmal AF & 11 & $100 \%$ & 34 & $72 \%$ & 0.055 \\
\hline Age [years] & 62 & $(57 ; 69)$ & 61 & $(56.5 ; 68.0)$ & 0.548 \\
\hline Body mass index & 25.73 & $(24.3 ; 28.7)$ & 27.28 & $(25 ; 31)$ & 0.475 \\
\hline Hypertension & 10 & $(91 \%)$ & 39 & $(83 \%)$ & \\
\hline Coronary artery disease & 1 & $(9 \%)$ & 1 & $(2 \%)$ & 0.346 \\
\hline Dilated/hypertrophic cardiomyopathy & 2 & $(18 \%)$ & 2 & $(4 \%)$ & 0.159 \\
\hline Diabetes mellitus & 2 & $(18 \%)$ & 1 & $(2 \%)$ & 0.089 \\
\hline GFR & 81.68 & $(74.3 ; 111.7)$ & 82.54 & $(71.2 ; 94.0)$ & 0.422 \\
\hline LVEF & 52 & $(47 ; 57)$ & 60 & $(56 ; 62)$ & 0.008 \\
\hline LA - short axis & 59 & $(58.0 ; 61.5)$ & 56 & $(51.5 ; 61.5)$ & 0.095 \\
\hline LA - long axis & 43 & $(40.5 ; 45.5)$ & 41 & $(38 ; 45)$ & 0.232 \\
\hline LA area & 24.94 & $(23.8 ; 27.1)$ & 22.96 & $(20.5 ; 26.7)$ & 0.036 \\
\hline Normalized LA area & 12.49 & $(11.8 ; 14.1)$ & 11.12 & $(10.0 ; 12.6)$ & 0.017 \\
\hline \multicolumn{6}{|l|}{ Procedural data } \\
\hline $\mathrm{PVI}+$ at least one additional lesion & 8 & $(73 \%)$ & 36 & $(77 \%)$ & 0.999 \\
\hline Roof line & 7 & $(64 \%)$ & 32 & $(68 \%)$ & 0.999 \\
\hline Mitral isthmus line & 7 & $(64 \%)$ & 29 & $(62 \%)$ & 0.999 \\
\hline Coronary sinus & 4 & $(36 \%)$ & 12 & $(26 \%)$ & 0.475 \\
\hline CFAE & 2 & $(18 \%)$ & 11 & $(23 \%)$ & 0.999 \\
\hline PVI + 1 lesion & 0 & & 7 & $(15 \%)$ & $x$ \\
\hline $\mathrm{PVI}+\geq 2$ lesions & 8 & $(73 \%)$ & 29 & $(62 \%)$ & 0.206 \\
\hline \multicolumn{6}{|l|}{ AAD in follow-up } \\
\hline AAD: class I & 1 & $(9 \%)$ & 8 & $(17 \%)$ & 0.671 \\
\hline AAD: class II & 9 & $(82 \%)$ & 38 & $(81 \%)$ & 0.999 \\
\hline AAD: class III & 3 & $(27 \%)$ & 11 & $(23 \%)$ & 0.999 \\
\hline Amiodarone & 2 & $(18 \%)$ & & $8(17 \%)$ & 0.999 \\
\hline Dronedarone & 1 & $(9 \%)$ & & $2(4 \%)$ & 0.444 \\
\hline Sotalol & 0 & & & $1(2.1 \%)$ & 0.999 \\
\hline
\end{tabular}

IQR — inter-quartile range; AF — atrial fibrillation; GFR — glomerular filtration rate; LVEF — left ventricular ejection fraction; LA — left atrium; $\mathrm{PVI}$ - pulmonary veins isolation; CFAE - complex fractionated electrograms; AAD - antiarrhythmic drugs

secondary LATAFL were observed in $14 \%$ and $9 \%$ of patients, respectively. Higher LATAFL recurrence rate was found only in patients with rheumatic heart disease [30]. Our data are comparable with reported total and late LATAFL recurrence (10\% and $8 \%$, respectively). Lower number of early LATAFL recurrence in our group is the result of our step-wise approach, focused on elimination of all peri-procedural arrhythmias, which was not applied by any of the previous authors describing early LATAFL recurrence.

\section{Types of LATAFL}

Nagamoto et al. [19] described primary (preprocedural) and secondary (procedural-related) LATAFL. Both types were further defined as early (which appeared during a procedure) and late (after a procedure) arrhythmias. He concluded that 
Table 3. Predictors of outcome after a single catheter ablation of atrial fibrillation ablation procedure.

\begin{tabular}{|c|c|c|c|c|}
\hline \multirow[t]{2}{*}{ A. Continuous variables (ROC analysis) } & \multirow[t]{2}{*}{ AUC } & \multicolumn{2}{|c|}{$95 \%$ confidence interval } & \multirow[t]{2}{*}{$\mathbf{P}$} \\
\hline & & Lower & Upper & \\
\hline History of atrial fibrillation & 0.441 & 0.357 & 0.525 & 0.192 \\
\hline Age & 0.564 & 0.474 & 0.654 & 0.156 \\
\hline Body mass index & 0.488 & 0.391 & 0.584 & 0.782 \\
\hline LA - long axis diameter in 4-chamber view & 0.651 & 0.572 & 0.730 & 0.001 \\
\hline LA - short axis diameter in 4-chamber view & 0.613 & 0.525 & 0.702 & 0.012 \\
\hline LA area & 0.646 & 0.569 & 0.723 & 0.001 \\
\hline Normalized LA area & 0.680 & 0.607 & 0.753 & 0.000 \\
\hline Left ventricular ejection fraction & 0.555 & 0.473 & 0.637 & 0.165 \\
\hline Renal function, GFR & 0.555 & 0.476 & 0.634 & 0.168 \\
\hline \multirow[t]{2}{*}{ B. Discrete variables } & \multirow[t]{2}{*}{ Hazard ratio } & \multicolumn{2}{|c|}{$95 \%$ confidence interval } & $\mathbf{P}$ \\
\hline & & Lower & Upper & \\
\hline Non paroxysmal atrial fibrillation & 2.568 & 1.354 & 4.872 & 0.003 \\
\hline Normalized LA > 11.5 & 2.435 & 1.410 & 4.206 & 0.002 \\
\hline Any additional lesion & 4.236 & 2.267 & 7.915 & 0.0001 \\
\hline Number of lesions* $\geq 2$ & 4.410 & 2.609 & 7.491 & 0.0001 \\
\hline \multirow[t]{2}{*}{ C. Multivariate Cox regression model } & \multirow[t]{2}{*}{ Hazard ratio } & \multicolumn{2}{|c|}{$95 \%$ confidence interval } & $\mathbf{p}$ \\
\hline & & Lower & Upper & \\
\hline Number of lesions ${ }^{*} \geq 2$ & 2.22 & 1.21 & 4.99 & 0.000 \\
\hline Enlarged LA, normalized LA > 11.5 & 1.88 & 1.11 & 3.19 & 0.019 \\
\hline
\end{tabular}

*Any performed additional lesions are included; GFR — glomerular filtration rate; LA — left atrium
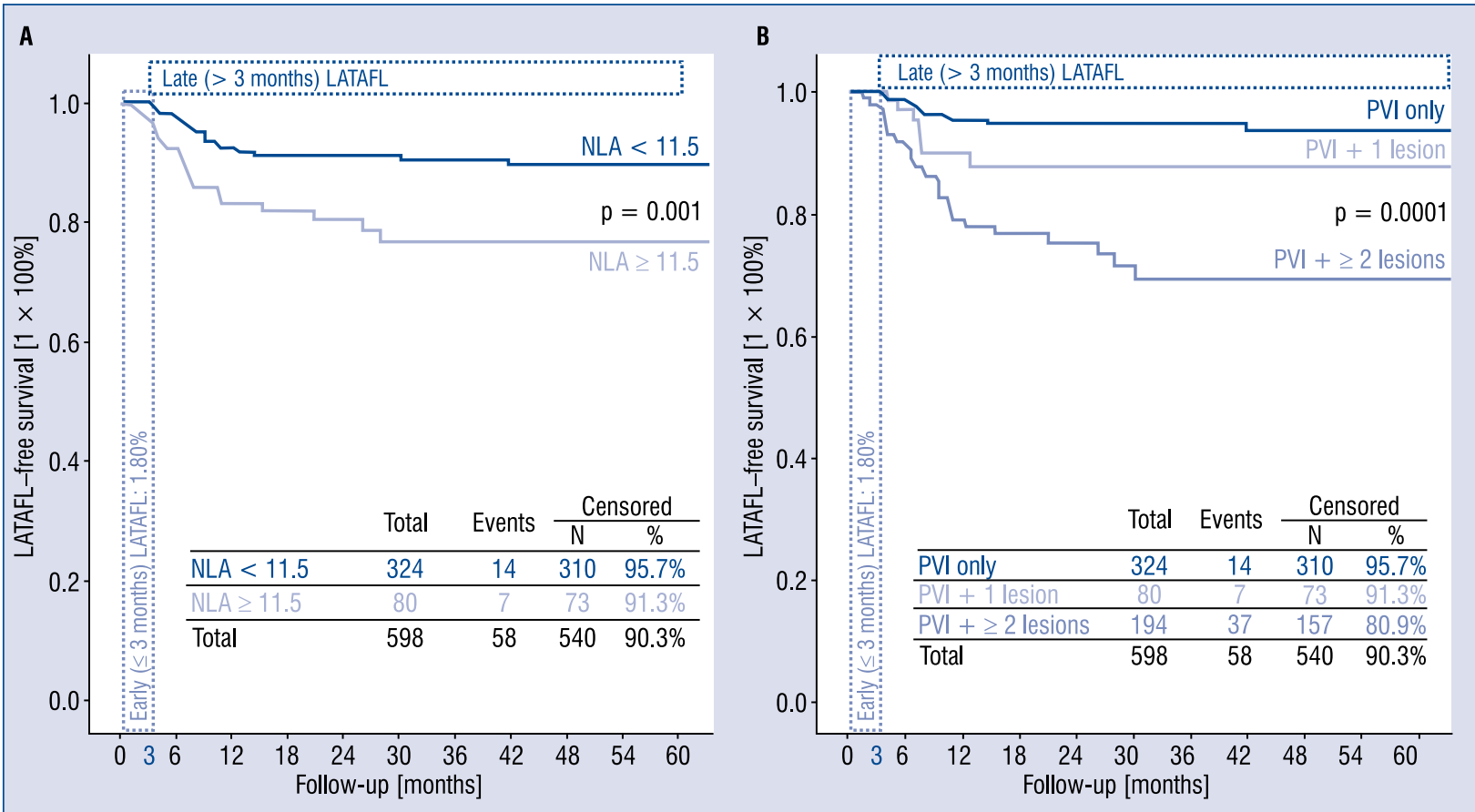

Figure 2. The size of left atrium (A), number of additional lesions (B) and a 5-year outcome. Kaplan-Meier event-free survival curve after a single catheter ablation; LATAFL — left atrial tachycardia and left atrial flutter; NLA — normalized left atrial area; $\mathrm{PVI}$ - pulmonary vein isolation. 
early secondary LATAFL should be ablated when it presented as a pre-ablation arrhythmia or a periprocedurally ongoing LATAFL resulting from a focal or gap-related macro-reentrant mechanism [19]. However, the ongoing LATAFL resulting from non-gap-related macro-reentry (except for typical isthmus-dependent atrial flutter) could be monitored [19].

Our definition of LATAFL follows recent consensus [4] and clearly demarcates peri-procedural (i.e. very early) from post-procedural (early, i.e. 3-month blanking period related, and late, i.e. $>3$ months since a procedure) LATAFL. Any periprocedural arrhythmia was approached according to our step-wise protocol. However, we cannot exclude that some of the observed LATAFLs, especially in patients after PVI only, were of the primary type (early or late), not identified before the procedure. Still, we performed a thorough selection of patients at inclusion, and no record of documented pre-procedural LATAFL was identified. Consequently, we focused on both early ( $\leq 3$ months) and late ( $>3$ months) LATAFLs only.

\section{Early secondary LATAFL}

We observed early secondary LATAFL only in patients with NPAF, lower left ventricular ejection fraction and enlarged LA. All these factors are related to structural remodeling of LA which results in local substrate prone to supra-ventricular arrhythmias (SVA) [31]. In all these cases we performed PVI and multiple additional lesions. We assume that such substrate modifications were enough for acute peri-procedural success, as we aimed for (following our protocol) total elimination of all ongoing SVA during the index procedure.

In an animal model, complete replacement of necrotic muscle with collagen was observed 4 weeks after radiofrequency ablation [32]. At 10 to 12 weeks, the replaced myocardium was less cellular and the collagen more dense. The necrotic atrial myocardium was replaced by a collagenous matrix containing small islands of surviving myocardium [32]. The anatomically remodeled atrium requires more time after ablation to reverse its vulnerability to triggers [33], and is more susceptible to early arrhythmia relapses [31]. Such a deep and wide injury within LA tissue needs time for local healing, i.e. the slow process of scarring which itself can be pro-arrhythmic. The delay in time between tissue injury to secondary LATAFL appearance could also be supported by the previous observation which showed that peri-CAAF inducibility of LATAFL does not predict its clinical recurrence in FU $[18,34]$. Still, we cannot exclude the coexistence of unknown pre-procedural or unmasked peri-procedural SVA. Interestingly, patients with early recurrence had shorter history of AF which further supported the idea of "healing-tissue"-related early LATAFL.

\section{Late secondary LATAFL}

Late secondary LATAFL recurrence was mainly observed in patients with a long history of AF, i.e. one of the mechanisms could be the continuous process of substrate remodeling within LA, which we hoped to slow down with CAAF, as curing could not be suspected in such a case. Subsequent explanation is the iatrogenic effect of any of the lesions produced, as well as gaps within linear lesions. Moreover, the interaction between the lesions and preexisting regions with abnormal electrical properties cannot be excluded. As all above are present in LA after CAAF, the final mechanism of secondary LATAFL is complex in its nature and difficult to be simply defined.

\section{Predictors of recurrence after CAAF}

While many predictors of AF recurrence following CAAF have been proposed [3, 4, 35], predictors of LATAFL are under debate. We show that independent predictors of LATAFL recurrence after CAAF are: the enlarged LA and multiple $(\geq 2)$ additional lesions performed during the index procedure.

Atrial enlargement, a result of atrial remodeling, is an important clinical predictor of AF maintenance [36]. The electrical remodeling in the atria, which results in shortening of the effective refractory period, is involved in the occurrence and maintenance of atrial fibrosis [37]. Contractile remodeling is evidenced by a decrease in atrial contractility which leads to dilation of the atria [38]. Structural remodeling within atria is evidenced by interstitial fibrosis and atrial dilatation [39]. This expanded the space between cardiomyocytes, likely due to the loss of cells and fibrotic replacement and expansion of the extracellular matrix, may also cause conduction delays between cardiomyocytes themselves and allow for alternate pathways of conduction [39]. These changes are electrically apparent as ectopic foci and anisotropic conduction, which create non-uniform wave fronts that allow abnormal re-entrant arrhythmias [40]. Atrial dilation increases the amount of atrial tissue that can accommodate reentry circuits [41]. Atrial dimensions are a particularly important determinant of the occurrence of multiple-circuit reentry [42]. 
Within larger atrial size more circuits can be accommodated and long-wavelength circuits that are too large for a normal-sized atrium can be supported [41]. Consequently, AF in the remodeled LA is not only trigger- but also substrate-dependent.

The iatrogenic effect of additional lesions is known [6-29]. Linear lesions result in linear scars which limit areas of LA activation. Nevertheless, performing linear lesions in dilated LA can be challenging due to the fact thatthey have to be longer than in not-dilated LA. The vast majority of arrhythmias that occur after CAAF are re-entrant (83\%) [1-6, 8-25] and use gaps in prior ablation lines. Elimination of the dominant arrhythmia may uncover, suppressed so far, ectopic foci. Additionally, electrogram-guided ablation and elimination of CFAE, aimed at ectopic foci, also result in many scattered areas of block, which in combination with existed regions of anisotropic conduction, promote substrate for re-entry. Consequently, the combination of LA enlargement and multiple lesions results in changed electrophysiological environment and development of secondary arrhythmias. Our stepwise approach showed that avoidance of multiple ablation lesions cannot be avoided in many patients. Nevertheless, the collected data show that limiting additional lesions (with step-wise approach), demonstration of conduction block within the lines and PV disconnection are likely to decrease the prevalence of post-CAAF LATAFL [21].

\section{Clinical implications}

Clinical implications of our results are substantial for patients planned for CAAF. Firstly, the outcome data should be presented. A procedure in patients with enlarged LA and premeditated for additional lesions might result in LATAFL. Consequently, subsequent CAAF can be needed. Secondly, thorough monitoring after CAAF is crucial. Incidence of late arrhythmia recurrence may be related to the extent of ECG monitoring and earlier recurrence may be missed in selected patients with no or minimal symptoms [4]. Clinical evaluation should be performed on regular basis and any complaint of "heart palpitations" [43] should be addressed. Finally, attention in control of patient-related risk factors remains an integral part of management after the CAAF procedure [4].

\section{Limitations of the study}

Our study has a few limitations: (1) This is a single-center, non-randomized report. However, there was no selection bias for study inclusion since all consecutive patients undergoing radiofrequency catheter ablation for $\mathrm{AF}$ at our institution were included for analysis. (2) A potential variability of operator experiences with the stepwise ablation approach might further limit our results. (3) We did not use any protocol of LATAFL induction after CAAF procedure. Nevertheless, it was previously shown that arrhythmia inducibility was not predictive of atrial arrhythmia recurrence in FU $[18,34]$. (4) According to the latest Consensus Report [4], the FU results are presented without consideration of recurrences during the blanking period. (5) The latest data [44], unknown at the time of selection of our study cohort, show that continuous monitoring with implanted devices is significantly superior to intermittent monitoring. Our FU was based on clinical evaluation and 7-day Holter-ECG recordings.

\section{Conclusions}

Our data show that a step-wise CAAF procedure results in relatively low LATAFL recurrence rate in a very long-term FU. Most LATAFL recurrences occurred in the first 12 months if only PVI was performed. In patients in whom any additional lesions were performed, most recurrences occurred in the first 30 months. A plateau in LATAFLfree survival was noted in both groups thereafter.

Higher rate of LATAFL recurrence was observed in patients with NPAF, enlarged LA and any additional lesions performed. We defined 2 independent predictors of LATAFL recurrence after CAAF: the enlarged LA and multiple $(\geq 2)$ additional lesions performed during the index procedure.

The presented data follow the latest indications for CAAF [4]. Performing CAAF at earlier stage of $\mathrm{AF}$, in not-dilated LA, may allow limiting the need for additional lesions. Such an approach is likely to decrease the prevalence of LATAFL after CAAF.

Conflicts of interest: Dr Maciej Wójcik was supported by European Heart Rhythm Association (2-year Clinical Electrophysiology Fellowship, 2007-2009).

\section{References}

1. Camm AJ, Lip GY, De Caterina R et al. 2012 focused update of the esc guidelines for the management of atrial fibrillation: An update of the 2010 esc guidelines for the management of atrial fibrillation. Developed with the special contribution of the european heart rhythm association. Eur Heart J, 2012; 33: 2719-2747.

2. Raviele A, Natale A, Calkins $\mathrm{H}$ et al. Venice chart international consensus document on atrial fibrillation ablation: 2011 update. J Cardiovasc Electrophysiol, 2012; 23: 890-923. 
3. Wójcik M, Berkowitsch A, Greiss H et al. Repeated catheter ablation of atrial fibrillation. Circ J, 2013; 77: 2271-2279.

4. Calkins H, Kuck KH, Cappato R et al. 2012 hrs/ehra/ecas expert consensus statement on catheter and surgical ablation of atrial fibrillation: Recommendations for patient selection, procedural techniques, patient management and follow-up, definitions, endpoints, and research trial design. Europace, 2012; 14: 528-606.

5. Berkowitsch A, Neumann T, Kuniss M et al. Therapy with reninangiotensin system blockers after pulmonary vein isolation in patients with atrial fibrillation: Who is a responder? Pacing Clin Electrophysiol, 2010; 33: 1101-1111.

6. Neumann T, Kuniss M, Erkapic D et al. Acute and long-term results of pvi at antrum using a novel high-density mapping catheter without help of $3 \mathrm{~d}$ electro-anatomic mapping in patients with paroxysmal and chronic atrial fibrillation. J Interv Card Electrophysiol, 2010; 27: 101-108.

7. Chugh A, Oral H, Lemola K et al. Prevalence, mechanisms, and clinical significance of macroreentrant atrial tachycardia during and following left atrial ablation for atrial fibrillation. Heart Rhythm, 2005; 2: 464-471.

8. Daoud EG, Weiss R, Augostini R et al. Proarrhythmia of circumferential left atrial lesions for management of atrial fibrillation. J Cardiovasc Electrophysiol, 2006; 17: 157-165.

9. Ouyang F, Antz M, Ernst S et al. Recovered pulmonary vein conduction as a dominant factor for recurrent atrial tachyarrhythmias after complete circular isolation of the pulmonary veins: Lessons from double lasso technique. Circulation, 2005; 111: 127-135.

10. Pappone C, Manguso F, Vicedomini G et al. Prevention of iatrogenic atrial tachycardia after ablation of atrial fibrillation: A prospective randomized study comparing circumferential pulmonary vein ablation with a modified approach. Circulation, 2004; 110: 3036-3042.

11. Shah D, Sunthorn H, Burri H et al. Narrow, slow-conducting isthmus dependent left atrial reentry developing after ablation for atrial fibrillation: ECG characterization and elimination by focal RF ablation. J Cardiovasc Electrophysiol, 2006; 17: 508-515.

12. Villacastin J, Perez-Castellano N, Moreno J, Gonzalez R. Left atrial flutter after radiofrequency catheter ablation of focal atrial fibrillation. J Cardiovasc Electrophysiol, 2003; 14: 417-421.

13. Deisenhofer I, Estner H, Zrenner B et al. Left atrial tachycardia after circumferential pulmonary vein ablation for atrial fibrillation: Incidence, electrophysiological characteristics, and results of radiofrequency ablation. Europace, 2006; 8: 573-582.

14. Ernst S, Ouyang F, Lober F, Antz M, Kuck KH. Catheter-induced linear lesions in the left atrium in patients with atrial fibrillation: An electroanatomic study. J Am Coll Cardiol, 2003; 42: 1271-1282.

15. Gerstenfeld EP, Callans DJ, Dixit S et al. Mechanisms of organized left atrial tachycardias occurring after pulmonary vein isolation. Circulation, 2004; 110: 1351-1357.

16. Hocini M, Sanders P, Jais P et al. Prevalence of pulmonary vein disconnection after anatomical ablation for atrial fibrillation: Consequences of wide atrial encircling of the pulmonary veins. Eur Heart J, 2005; 26: 696-704.

17. Karch MR, Zrenner B, Deisenhofer I et al. Freedom from atrial tachyarrhythmias after catheter ablation of atrial fibrillation: A randomized comparison between 2 current ablation strategies. Circulation, 2005; 111: 2875-2880.

18. Leong-Sit P, Robinson M, Zado ES et al. Inducibility of atrial fibrillation and flutter following pulmonary vein ablation. J Cardiovasc Electrophysiol, 2013; 24: 617-623.
19. Nagamoto Y, Tsuchiya T, Miyamoto K, Yamaguchi T, Takahashi N. Atrial tachycardia during ongoing atrial fibrillation ablation. Ensite array analysis. Circ J, 2011; 75: 1080-1089.

20. Sawhney N, Anousheh R, Chen W, Feld GK. Circumferential pulmonary vein ablation with additional linear ablation results in an increased incidence of left atrial flutter compared with segmental pulmonary vein isolation as an initial approach to ablation of paroxysmal atrial fibrillation. Circulation Arrhythmia Electrophysiol, 2010; 3: 243-248.

21. Chae S, Oral H, Good E et al. Atrial tachycardia after circumferential pulmonary vein ablation of atrial fibrillation: Mechanistic insights, results of catheter ablation, and risk factors for recurrence. J Am Coll Cardiol, 2007; 50: 1781-1787.

22. Chang SL, Tsao HM, Lin YJ et al. Differentiating macroreentrant from focal atrial tachycardias occurred after circumferential pulmonary vein isolation. J Cardiovasc Electrophysiol, 2011; 22: 748-755.

23. Cummings JE, Schweikert R, Saliba $W$ et al. Left atrial flutter following pulmonary vein antrum isolation with radiofrequency energy: Linear lesions or repeat isolation. J Cardiovasc Electrophysiol, 2005; 16: 293-297.

24. Jais P, Hocini M, Hsu LF et al. Technique and results of linear ablation at the mitral isthmus. Circulation, 2004; 110: 2996-3002.

25. Kanagaratnam L, Tomassoni G, Schweikert R et al. Empirical pulmonary vein isolation in patients with chronic atrial fibrillation using a three-dimensional nonfluoroscopic mapping system: Long-term follow-up. Pacing Clin. Electrophysiol, 2001; 24: 1774-1779.

26. Oral H, Scharf C, Chugh A et al. Catheter ablation for paroxysmal atrial fibrillation: Segmental pulmonary vein ostial ablation versus left atrial ablation. Circulation, 2003; 108: 2355-2360.

27. Oral H, Chugh A, Lemola K et al. Noninducibility of atrial fibrillation as an end point of left atrial circumferential ablation for paroxysmal atrial fibrillation: A randomized study. Circulation, 2004; 110: 2797-2801.

28. Rostock T, Drewitz I, Steven D et al. Characterization, mapping, and catheter ablation of recurrent atrial tachycardias after stepwise ablation of long-lasting persistent atrial fibrillation. Circulation Arrhythmia Electrophysiology, 2010; 3: 160-169.

29. Sotomi $\mathrm{Y}$, Inoue $\mathrm{K}$, Ito $\mathrm{N}$ et al. Cause of very late recurrence of atrial fibrillation or flutter after catheter ablation for atrial fibrillation. Am J Cardiol, 2013; 111: 552-556.

30. Wang XH, Huang CX, Liu X et al. Ablation of atrial tachycardia occurring after catheter ablation of atrial fibrillation in patients with corrected rheumatic valve disease. J Interv Card Electrophysiol, 2012; 35: 45-56.

31. Bertaglia E, Stabile G, Senatore G et al. Predictive value of early atrial tachyarrhythmias recurrence after circumferential anatomical pulmonary vein ablation. Pacing Clin Electrophysiol, 2005; 28: 366-371.

32. Taylor GW, Kay GN, Zheng X, Bishop S, Ideker RE. Pathological effects of extensive radiofrequency energy applications in the pulmonary veins in dogs. Circulation, 2000; 101: 1736-1742.

33. Allessie MA. Atrial electrophysiologic remodeling: Another vicious circle? J Cardiovasc Electrophysiol, 1998; 9: 1378-1393.

34. Kurotobi T, Shimada Y, Kino N et al. Inducible atrial tachycardias with multiple circuits in a stepwise approach are associated with increased episodes of atrial tachycardias after catheter ablation. J Electrocardiol, 2012; 45: 102-108.

35. Wojcik M, Erkapic D, Berkowitsch A et al. Ipsilateral circumferential radiofrequency ablation of atrial fibrillation with irrigated tip catheter. Circ J, 2013; 77: 2280-2287. 
Cardiology Journal 2015, Vol. 22, No. 5

36. Henry WL, Morganroth J, Pearlman AS et al. Relation between echocardiographically determined left atrial size and atrial fibrillation. Circulation, 1976; 53: 273-279.

37. Van Wagoner DR. Electrophysiological remodeling in human atrial fibrillation. Pacing Clin Electrophysiol, 2003; 26: 1572-1575.

38. Schotten U, de Haan S, Neuberger HR et al. Loss of atrial contractility is primary cause of atrial dilatation during first days of atrial fibrillation. Am J Physiol Heart Circ Physiol, 2004; 287: H2324-2331.

39. Pellman J, Lyon RC, Sheikh F. Extracellular matrix remodeling in atrial fibrosis: Mechanisms and implications in atrial fibrillation. J Mol Cell Cardiol, 2010; 48: 461-467.

40. Eckstein J, Verheule S, de Groot NM, Allessie M, Schotten U. Mechanisms of perpetuation of atrial fibrillation in chronically dilated atria. Prog Biophys Mol Biol, 2008; 97: 435-451.
41. Nattel S, Burstein B, Dobrev D. Atrial remodeling and atrial fibrillation: Mechanisms and implications. Circulation Arrhythmia Electrophysiol, 2008; 1: 62-73.

42. Zou R, Kneller J, Leon LJ, Nattel S. Substrate size as a determinant of fibrillatory activity maintenance in a mathematical model of canine atrium. Am J Physiol Heart Circ Physiol, 2005; 289: H1002-H1012.

43. Neumann T, Erdogan A, Dill T et al. Asymptomatic recurrences of atrial fibrillation after pulmonary vein isolation. Europace, 2006; 8: 495-498.

44. Charitos EI, Stierle U, Ziegler PD et al. A comprehensive evaluation of rhythm monitoring strategies for the detection of atrial fibrillation recurrence: Insights from 647 continuously monitored patients and implications for monitoring after therapeutic interventions. Circulation, 2012; 126: 806-814. 